Article

\title{
Effect of Rotor Spacing and Duct Diffusion Angle on the Aerodynamic Performances of a Counter-Rotating Ducted Fan in Hover Mode
}

\author{
Woo-Yul Kim, Santhosh Senguttuvan $\mathbb{D}$ and Sung-Min Kim * \\ School of Mechanical Engineering, Sungkyunkwan University, 300 Cheoncheon-dong, Suwon 16419, Korea; \\ samsun215@skku.edu (W.-Y.K.); santhosh@skku.edu (S.S.) \\ * Correspondence: smkim@skku.edu; Tel.: +82-31-290-7433
}

Received: 21 August 2020; Accepted: 20 October 2020; Published: 23 October 2020

\begin{abstract}
The aerodynamic performance of a counter-rotating ducted fan in hover mode is numerically analyzed for different rotor spacings and duct diffusion angles. The design of the counter-rotating fan is inspired by a custom-designed single rotor ducted fan used in a previous study. The numerical model to predict the aerodynamic performance of the counter-rotating ducted fan is developed by adopting the frozen rotor approach for steady-state incompressible flow conditions. The relative angle between the front and the rear rotor is examined due to the usage of the frozen rotor model. The results show that the variation of thrust for the different relative angles is extremely low. The aerodynamic performances are evaluated by comparing the thrust, thrust coefficient, power coefficient, and figure of merit (FOM). The thrust, thrust coefficient, and FOM slightly increase with increasing rotor spacing up to $200 \mathrm{~mm}$, regardless of the duct diffusion angle, and reduce on further increase in the rotor spacing. The duct diffusion angle of $0^{\circ}$ generates about $9 \%$ higher thrust and increases the FOM by $6.7 \%$, compared with the $6^{\circ}$ duct diffusion angle. The duct diffusion angle is highly effective in improving the thrust and FOM of the counter-rotating ducted fan, rather than the rotor spacing.
\end{abstract}

Keywords: thrust coefficient; power coefficient; figure of merit; frozen rotor; UAV

\section{Introduction}

Unmanned aerial vehicles (UAV) are of global interest, since they can perform versatile tasks in the military, search and rescue, agriculture, and transportation fields. Particularly, rotary wing UAVs with vertical take-off and landing (VTOL) propulsion systems are highly desired due to high maneuverability and the ability to take-off and land vertically. In rotary wing UAVs, a ducted fan where a duct surrounds the rotors is more efficient in producing thrust than an open rotor [1]. According to actuator disk theory, the duct reduces the blade tip loss, enabling the ducted fan to double the thrust [2]. The duct also reduces the rotor noise and protects the high-speed rotors from the external environment.

The design of the duct plays a vital role in improving the aerodynamic performance of the ducted fan. One of the key considerations in the design of the ducted fan is the duct profile. The duct profile is usually based on airfoil shapes, due to aerodynamical advantage. Yilmaz et al. [3] experimentally studied the aerodynamic performance of a ducted fan in hover mode for five different duct profiles, based on National Advisory Committee for Aeronautics (NACA) airfoils. Pressure distributions on the inner surface of the duct and velocity profiles at both the duct inlet and outlet planes were investigated for the five different duct profiles. It was found that the propulsive efficiency was significantly affected by the duct profile. Bontempo and Manna [4] numerically investigated the effect of the camber and thickness of the duct profile on the aerodynamic performance of ducted fans. They found that the propulsive efficiency of the duct increased with the increase in the camber and thickness of the 
duct profile. $\mathrm{Xu}$ et al. [5] developed an asymmetrical duct with a rounded and enlarged leading edge. The leading edge is designed as inflatable so that it can inflate and retract when needed [6,7]. The inflated leading edge is designed to encounter the flow separation that typically occurs around the inner surface of the duct when the ducted fan transits from forward flight (crosswind) to hovering, or from hovering to forward flight. The developed duct model eliminates the flow separation at the crosswind conditions of a $30 \mathrm{~m} / \mathrm{s}$ velocity at a $50^{\circ}$ angle of attack. Although the proposed duct model was suitable to deal with the flow separation, it involved certain complications concerned with the practical implementation of the inflatable material in ducted-fan UAVs. Graf et al. [8] conducted experiments to examine the aerodynamic characteristics of a ducted fan for five duct profiles with different leading edge radius of curvatures and duct wall thicknesses, both in hover and forward flight conditions. As a result, the characteristics of the flow separation on the leading edge of the duct in hover and forward flight modes were found to be different. The duct profile that produced the best performance was different for the hover and forward flight modes.

Apart from duct design, a counter-rotating system, where two rotors rotate in the opposite direction on the same axis, can increase the thrust and aerodynamic performance of a ducted fan. The counter-rotating system has been successfully applied to wind turbines [9,10], axial flow pumps [11,12], axial fans [13,14], and propellers [15,16]. A key advantage of this system is that it can produce a higher thrust than a single rotor, and the torque produced by the two rotors is canceled by each other. The essential parameters affecting the aerodynamic performance in the counter-rotating fan are tip clearance and rotor spacing. Ryu et al. [17] examined the effect of the tip clearance of the front and rear rotors on the aerodynamic performances of the counter-rotating ducted fan in hover mode. In the counter-rotating ducted system, the thrust is affected by relative tip clearances of the front and rear rotors. It was found that, in order to improve the aerodynamic performance of the counter-rotating ducted fan, the tip clearance need not be a minimum for both the front and rear rotors. Among various tip clearance configurations in the study, the configuration with a small tip clearance in the front rotor and a large tip clearance in the rear rotor produced the highest thrust coefficient. Moreover, the total thrust of the counter-rotating ducted fan was significantly affected by the tip clearance of the rear rotor. Han et al. [18] conducted experiments and simulations to investigate the aerodynamic performances of a counter-rotating fan with and without ducts for different blade pitch angles, rotor spacings, and tip clearances in hover mode. The results showed that the counter-rotating fan with a duct generated more thrust than the open counter-rotating fan because of the negative pressure region around the duct's leading edge created by the duct. They also found that as the rotor spacing increased, the thrust coefficient and figure of merit of the counter-rotating fan, both with and without the duct, increased. However, when the rotor spacing became higher than the rotor radius, both the thrust coefficient and figure of merit became constant.

Numerical modeling of the counter-rotating fan necessitates the consideration of the relative motion between the two rotors. This relative motion can be modeled by either a mixing plane, a frozen rotor, or a moving mesh approach. Of the three methods, the moving mesh method produces accurate, realistic flow physics by employing the unsteady coupling of the rotors. However, the moving mesh approach requires high computational effort, resulting in researchers and industrial fan designers opting for either the mixing plane or the frozen rotor approach. Besides that, for steady-state simulations, the mixing plane and frozen rotor methods produce reasonably accurate results. A major disadvantage of the mixing plane approach is that it is not capable of predicting the effect of wakes from the top rotor on the downstream rotor of the counter-rotating fan system. Compared to the mixing plane approach, the frozen rotor approach calculates the non-uniform circumferential velocity and pressure distributions to accurately predict the wake mixing in the downstream rotor and rotor-to-rotor flow physics [19].

Despite several studies conducted to enhance the aerodynamic performance of UAVs, there is still scope for improvement. It is highly important to further study and improve the aerodynamic performance of UAVs. In the present study, the effects of rotor spacing and the duct diffusion angle on the aerodynamic performances of a counter-rotating ducted fan in hover mode are investigated 
numerically using the frozen rotor approach. A ducted fan model, designed by Akturk and Camci [20], is used for the present numerical study due to readily available detailed experimental data. Three essential aerodynamic performance parameters - thrust coefficient, power coefficient, and figure of merit-are examined for different rotor spacings and duct diffusion angles.

\section{Numerical Model}

\subsection{Model Description}

Figure 1 shows the schematic of the three-dimensional counter-rotating ducted fan based on the fan and duct models of Akturk and Camci [20], where they studied a single ducted fan. The design of both of the fans in the present study was identical to the single fan used by Akturk and Camci [20]. The duct diameter was $559 \mathrm{~mm}$, and the fan had eight blades. The two rotors (fans) had the same tip clearance of $1.71 \%$ and rotated in opposite directions. Table 1 summarizes the detailed dimensions of the counter-rotating ducted fan. The rotor spacing (s) was changed from $120 \mathrm{~mm}$ to $240 \mathrm{~mm}$ in increments of $40 \mathrm{~mm}$. While changing the rotor spacing, the length of duct was also equally changed to maintain the length of the duct diffuser at $117.85 \mathrm{~mm}$. Two duct diffusion angles $(\theta)$ of $0^{\circ}$ and $6^{\circ}$ were considered.
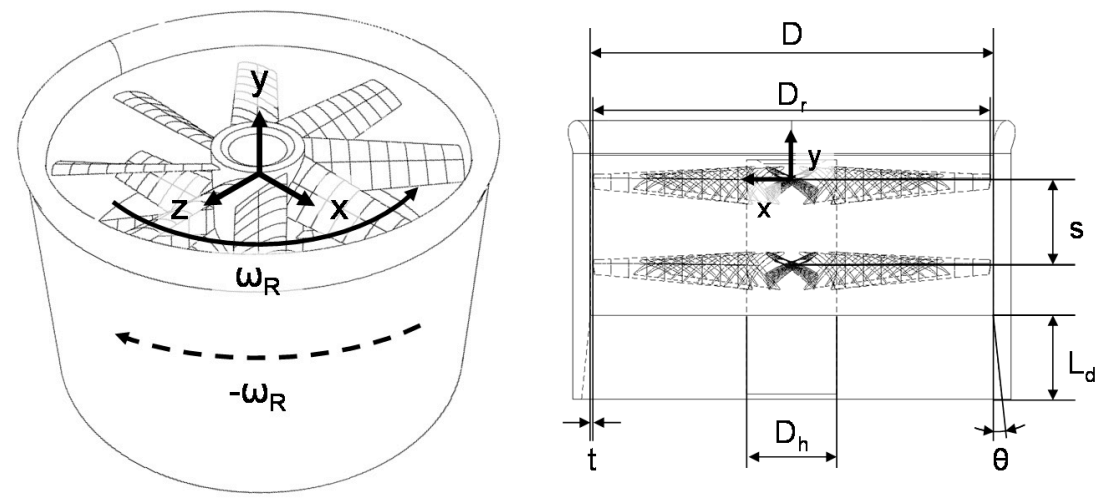

Figure 1. Schematic of counter-rotating ducted fan.

Table 1. Counter-rotating ducted fan dimensions.

\begin{tabular}{cc}
\hline Parameter & Value \\
\hline Duct diameter, $\mathrm{D}$ & $559.0 \mathrm{~mm}$ \\
Rotor diameter, $\mathrm{D}_{\mathrm{r}}$ & $558.8 \mathrm{~mm}$ \\
Tip clearance, $\mathrm{t}$ & $1.71 \%$ \\
Hub diameter, $\mathrm{D}_{\mathrm{h}}$ & $127 \mathrm{~mm}$ \\
Length of the duct diffuser, $\mathrm{L}_{\mathrm{d}}$ & $117.85 \mathrm{~mm}$ \\
\hline
\end{tabular}

\subsection{Governing Equation}

The three-dimensional numerical model to predict the aerodynamic performances of the counter-rotating ducted fan in hover mode was developed using the commercial Computational Fluid Dynamics (CFD) software Ansys CFX 19.1 [21]. The Reynolds-averaged Navier-Stokes (RANS) equations for steady-state, turbulent, and incompressible flow conditions with constant properties were used as the governing equations. The time-averaged continuity and momentum [21] are expressed as follows:

Continuity equation:

$$
\frac{\partial\left(\rho u_{j}\right)}{\partial x_{i}}=0
$$


Momentum equation for the stationary domain:

$$
\frac{\partial\left(\rho u_{j}\right)}{\partial x_{i}}=-\frac{\partial P}{\partial x_{i}}+\mu \frac{\partial^{2} u_{i}}{\partial x_{j}^{2}}+\rho g_{i}
$$

Momentum equation for the rotating domain:

$$
\frac{\partial\left(\rho u_{j}\right)}{\partial x_{i}}=-\frac{\partial P}{\partial x_{i}}+\mu \frac{\partial^{2} u_{i}}{\partial x_{j}^{2}}+\rho g_{i}+S_{M}
$$

where the source term, $S_{M}$, includes the centrifugal force and Coriolis force for the rotating reference frame, which is expressed as

$$
S_{M}=-\rho\left[2 \overrightarrow{\omega_{R}} \times \vec{u}+\overrightarrow{\omega_{R}} \times\left(\overrightarrow{\omega_{R}} \times \vec{r}\right)\right]
$$

In the present study, the shear stress transport (SST) k- $\omega$ turbulent model developed by Menter [22,23] was used to solve the turbulent flow field. As per the SST k- $\omega$ turbulence model, the boundary layer was calculated by the standard $k-\omega$ turbulence model, and the freestream region was calculated by the $k-\varepsilon$ turbulence model, which was incorporated by applying a blending function. The SST k- $\omega$ turbulence model accurately predicts the adverse pressure gradient and flow separation at the wall $[22,23]$. The turbulent kinetic energy and dissipation rate are expressed as follows:

Turbulent kinetic energy equation:

$$
\frac{\partial\left(\rho u_{j} k\right)}{\partial x_{k}}=P-\beta^{*} \rho \omega k+\frac{\partial}{\partial x_{j}}\left[\left(\mu+\sigma_{k} \mu_{t}\right) \frac{\partial k}{\partial x_{j}}\right]
$$

Dissipation rate equation:

$$
\frac{\partial\left(\rho u_{j} \omega\right)}{\partial x_{j}}=\frac{\gamma}{v_{t}} P-\beta \rho \omega^{2}+\frac{\partial}{\partial x_{j}}\left[\left(\mu+\omega_{\omega} \mu_{t}\right) \frac{\partial k}{\partial x_{j}}\right]+2\left(1-F_{1}\right) \frac{\rho \sigma_{\omega 2}}{\omega} \frac{\partial k}{\partial x_{j}} \frac{\partial \omega}{\partial x_{j}}
$$

The production limiter $(P)$ in Equation (5) is expressed as

$$
P=\tau_{i j} \frac{\partial u_{i}}{\partial x_{j}}
$$

where

$$
\begin{gathered}
\tau_{i j}=\mu_{t}\left(2 S_{i j}-\frac{2}{3} \frac{\partial u_{k}}{\partial x_{k}} \delta_{i j}\right)-\frac{2}{3} \rho k \delta_{i j} \\
S_{i j}=\frac{1}{2}\left(\frac{\partial u_{i}}{\partial x_{j}}+\frac{\partial u_{j}}{\partial x_{i}}\right)
\end{gathered}
$$

and the turbulent eddy viscosity is expressed as

$$
\mu_{t}=\frac{\rho a_{1} k}{\max \left(a_{1} \omega, \Omega F_{2}\right)}
$$

Let $\phi_{1}$ represent the constants $\sigma_{k 1}, \sigma_{\omega 1}$, and $\beta_{1}$ in the k- $\omega$ turbulence model, $\phi_{2}$ represent the constants $\sigma_{k 2}, \sigma_{\omega 2}$, and $\beta_{2}$ in k- $\varepsilon$ turbulence model, and $\phi$ represent the constants $\sigma_{k}, \sigma_{\omega}$, and $\beta$ in the k- $\omega$ SST turbulence model, respectively. The relationship between $\left.\phi_{1}\right] \phi_{1}$, and $\phi_{2}$ is expressed as

$$
\phi=F_{1} \phi_{1}+\left(1-F_{1}\right) \phi_{2}
$$


The additional functions in the SST k- $\omega$ turbulence model are as follows:

$$
\begin{gathered}
F_{1}=\tanh \left(\arg _{1}^{4}\right) \\
\arg _{1}=\min \left[\max \left(\frac{\sqrt{k}}{\beta^{*} \omega d}, \frac{500 v}{d^{2} \omega}\right), \frac{4 \rho \sigma_{\omega 2} k}{C D_{k \omega} d^{2}}\right] \\
C D_{k \omega}=\max \left(2 \rho \sigma_{\omega 2} \frac{1}{\omega} \frac{\partial k}{\partial x_{j}} \frac{\partial \omega}{\partial x_{j}}, 10^{-20}\right) \\
F_{2}=\tanh \left(\arg _{2}^{2}\right) \\
\arg _{2}=\max \left(2 \frac{\sqrt{k}}{\beta^{*} \omega d}, \frac{500 v}{d^{2} \omega}\right)
\end{gathered}
$$

where $d$ is the distance from the field point to the nearest wall and $\Omega=\sqrt{2 W_{i j} W_{i j}}$, with

$$
W_{i j}=\frac{1}{2}\left(\frac{\partial u_{i}}{\partial x_{j}}-\frac{\partial u_{j}}{\partial x_{i}}\right)
$$

All the constants are as follows:

$$
\begin{gathered}
\gamma_{1}=\frac{\beta_{1}}{\beta^{*}}-\frac{\sigma_{\omega 1} \kappa^{2}}{\sqrt{\beta^{*}}}, \gamma_{2}=\frac{\beta_{2}}{\beta^{*}}-\frac{\sigma_{\omega 2} \kappa^{2}}{\sqrt{\beta^{*}}}, \sigma_{k 1}=0.85, \sigma_{\omega 1}=0.5, \beta_{1}=0.075, \sigma_{k 2}=1.0, \\
\sigma_{\omega 2}=0.856, \beta_{2}=0.0828, \beta^{*}=0.09, \kappa=0.41, a_{1}=0.31 .
\end{gathered}
$$

\subsection{Numerical Method}

The numerical simulations were performed as three-dimensional with steady-state and incompressible flow assumptions. Figure 2 shows the grid system, boundary, and interface conditions of the counter-rotating ducted fan. The computational domain consisted of a stationary domain, surrounding the duct and part of the hub, and two rotating domains surrounding each rotor and the remaining part of the hub. The unstructured grid was used for both the stationary and the rotating domain, and the structured hexahedral grid was used for the external fluid domain. The dimensionless distance from the wall to the first node of the mesh, defined as $y+$, is crucial in the SST k- $\omega$ turbulence model. The inflation layers were used to achieve a y+ value less than 2.5 along all the walls to capture fluid interactions in the viscous sublayer. The axial length of the computational domain was $3.0 \mathrm{D}$ and $6.0 \mathrm{D}$ from the origin, located at the center of the front rotor, and the radial length was $2.5 \mathrm{D}$ from the $y$-axis. The multiple reference frame (MRF) method was used to simulate the rotation of the rotors. The rotating domain was considered to rotate relative to the stationary domain by adding the centrifugal force and Coriolis force (see Equations (2) and (3)). The frozen rotor, mixing plane (stage), and sliding mesh methods were the available methods to model the interface between rotating and stationary domains in the MRF method. In the present study, the frozen rotor method was used for the interface between the rotating and stationary domains, as well as the interface between the two rotating domains. When using the frozen rotor, the rotor in the rotating domain is considered to be in a frozen state; this means the rotor position is fixed relative to the stationary domain. In the frozen rotor method, the results may vary depending on the rotor position. Hence, in the present study, the positions of the front and rear rotors were tested for four relative angles: $0^{\circ}, 11.25^{\circ}, 22.5^{\circ}$, and $33.75^{\circ}$. The opening boundary condition with zero-gauge pressure was applied to the external surfaces of the fluid domain to allow inward or outward airflow in hover mode. The no-slip condition was applied to the walls of the duct, hub, and rotors, except for the inner wall, the duct adjacent to the rotating domains. The counter-rotating wall boundary condition with an equal velocity in the opposite direction corresponding to the rotating domain was applied to the inner wall of the duct. 
To reduce the computational cost, only one-eighth of the counter-rotating ducted fan was modeled in the computational domain by applying the periodic boundary conditions to the side surfaces.

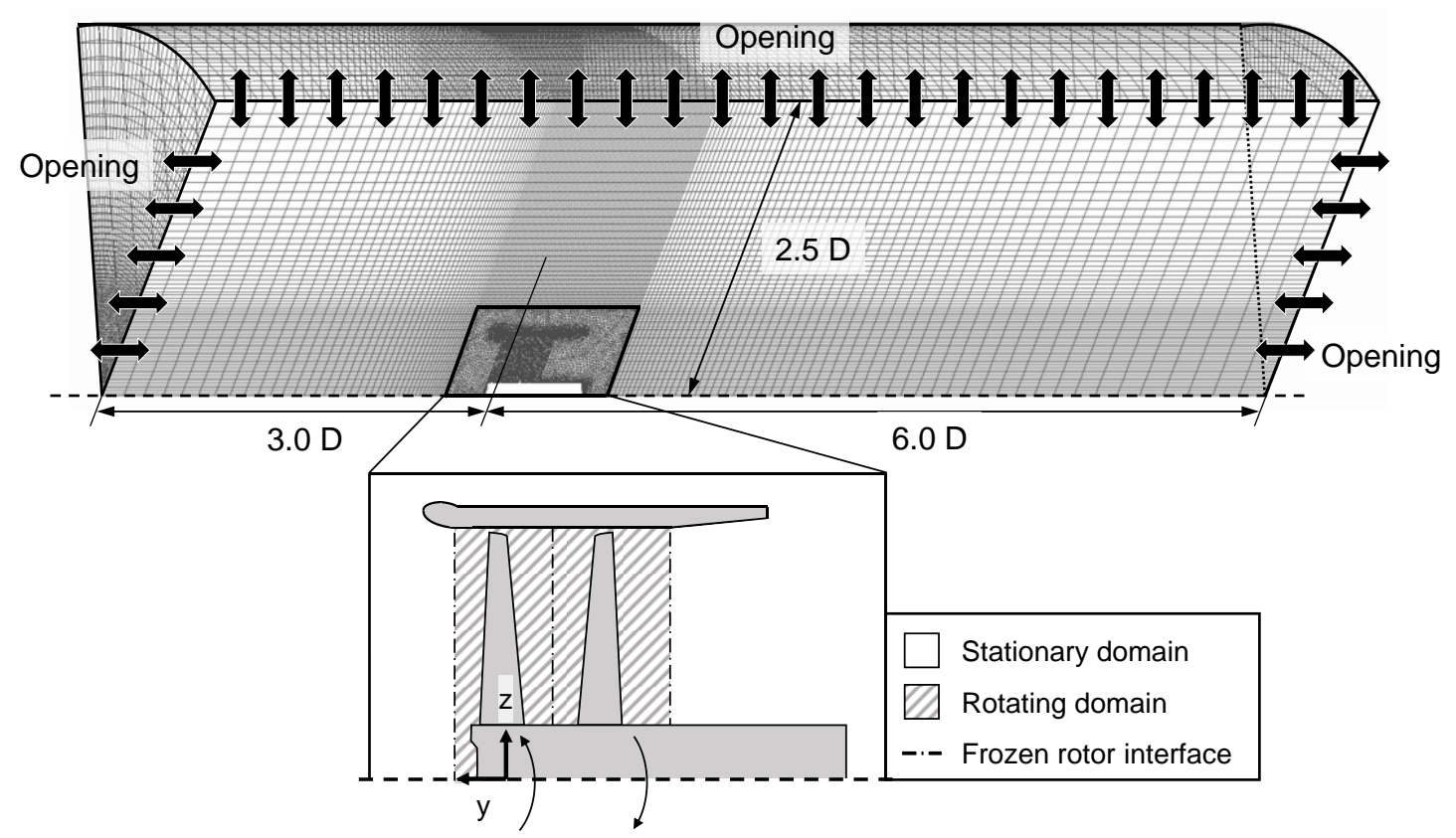

Figure 2. The grid system, boundary, and interface conditions of the counter-rotating ducted fan.

\subsection{Grid Independence Test and Validation}

The aerodynamic performance parameters, thrust coefficient, power coefficient, and figure of merit are expressed as follows:

Thrust coefficient:

$$
C_{T}=\frac{\text { Thrust }}{\rho N^{2} D^{4}}
$$

Power coefficient:

$$
C_{P}=\frac{\text { Power }}{\rho N^{3} D^{5}}
$$

Figure of merit (FOM):

$$
F O M=\frac{C_{T}^{3 / 2}}{\sqrt{2} C_{P}}
$$

The single ducted fan model of Akturk and Camci [20] was simulated to conduct grid independence testing and validation. The grid independence test was conducted using four different grid systems comprising $3.98,4.79,5.87$, and 7.02 million cells at $1500 \mathrm{rpm}$. The thrust coefficient of the different grid systems is compared in Figure 3a. The discrepancy in the thrust coefficient among the 4.79, 5.87, and 7.02 million cell grids was less than $1.0 \%$, and, thus, the grid system with 4.79 million cells was selected. The total simulation time for the selected mesh size was about $10 \mathrm{~h}$ in a machine with an Intel Core i7 6700 (4 physical cores and 8 threads). For validation, the single ducted fan was simulated under six different rotational speeds, corresponding to the experiments of Akturk and Camci [20]. Figure $3 \mathrm{~b}$ compares the thrust generated for the six different rotational speeds in the present numerical study with the experiment and numerical results of Akturk and Camci [20]. The present numerical results show good agreement with the results of Akturk and Camci [20]. 


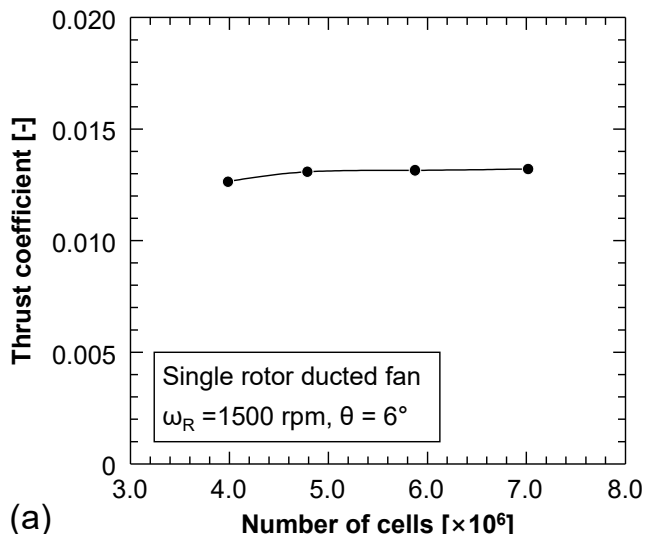

Figure 3. (a) The thrust coefficient for different grid systems, and (b) comparison of thrust on single rotor ducted fan of the present numerical study with the experiment and numerical results of Akturk and Camci [20].

\section{Results and Discussion}

\subsection{Effect of Relative Angle between Front and Rear Rotor}

Initially, the effect of the relative angle between the front and rear rotor was tested for four relative angles: $0^{\circ}, 11.25^{\circ}, 22.5^{\circ}$, and $33.75^{\circ}$. Each relative angle configuration was simulated for three rotational speeds-1500 rpm, $2100 \mathrm{rpm}$, and $2700 \mathrm{rpm}$-and two rotor spacings of $120 \mathrm{~mm}$, and $200 \mathrm{~mm}$. The diffusion angle of the duct for all of the relative angle configurations was $6^{\circ}$. Figure 4 shows the thrust generated at each relative angle configuration for the different rotational speeds and rotor spacings. The thrust was not significantly affected by the relative angle at $1500 \mathrm{rpm}$ for both rotor spacings. However, minor variations in the thrust were observed as the rotational speed increased for both rotor spacings.

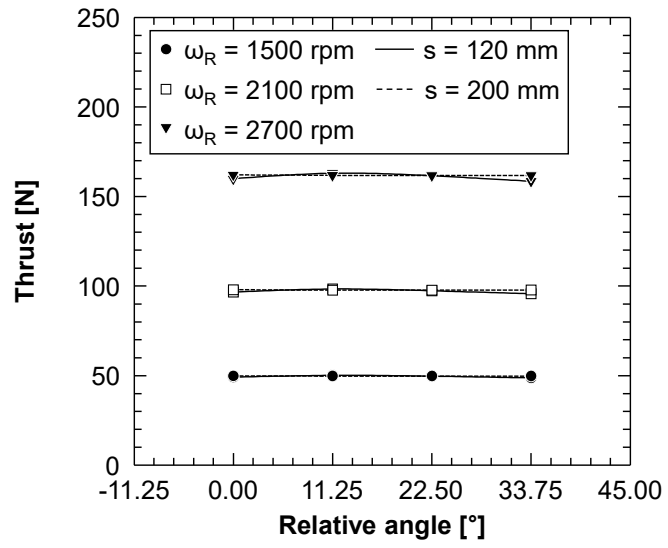

Figure 4. Thrust generated at four relative angles between the front and rear rotors for different rotational speeds and rotor spacings.

Figure 5a-d shows the total pressure contours at Plane 3 for the different relative angles under the conditions of a $2700 \mathrm{rpm}$ rotational speed and $200 \mathrm{~mm}$ rotor spacing. Refer to Figure 6 for the location of Plane 3 in the domain. The pressure contours of the $2700 \mathrm{rpm}$ rotational speed and $200 \mathrm{~mm}$ rotor spacing cases were compared since the thrust was slightly affected by the relative angles as the rotational speed increased. The high-pressure region, widely distributed across the center of the interface, shifted periodically with the change in relative angle. The difference in the pressure distribution for the different relative angles was negligible. Hence, the effect of the relative angle between the front and rear rotor was negligible, and the $0^{\circ}$ relative angle was considered for 
further simulations to evaluate the aerodynamic performance of the counter-rotating ducted fan. Similar relative angle studies conducted in a gas turbine pre-swirl system [24], an axial turbine [25], and a centrifugal pump [26] also concluded that the effect of the relative angle is negligible.

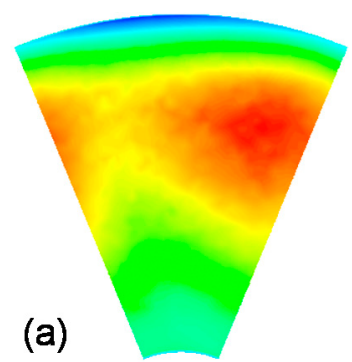

(b)
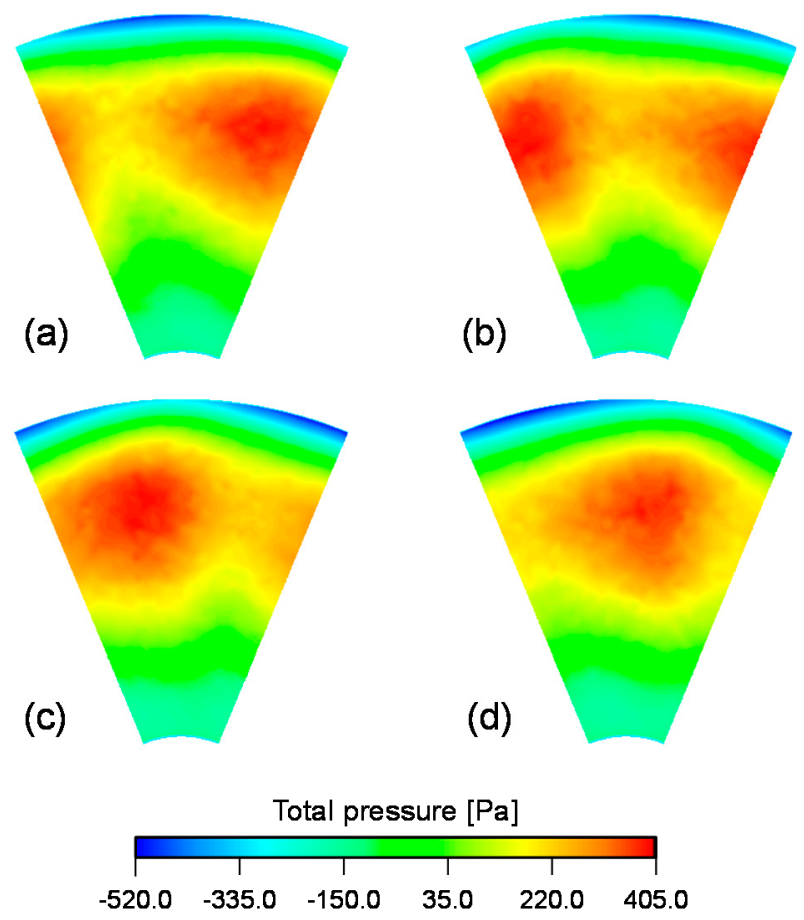

Figure 5. Total pressure contour and four relative angles between the front and rear rotors of (a) $0^{\circ}$; (b) $11.25^{\circ}$; (c) $22.5^{\circ}$; and (d) $33.75^{\circ}$ at Plane 3 , when $\mathrm{s}=200 \mathrm{~mm}, \theta=6^{\circ}$, and $\omega \mathrm{R}=2700 \mathrm{rpm}$. Refer to Figure 6 for the location of Plane 3.

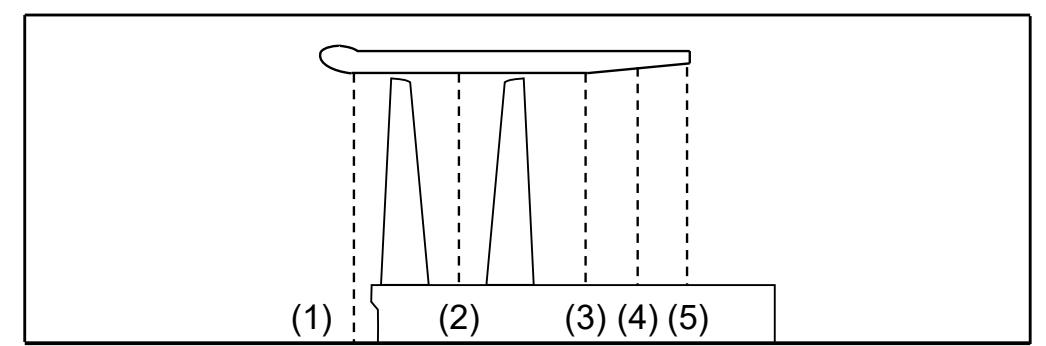

(1) Plane 1 - Interface plane between front rotating domain and stationary domain

(2) Plane 2 - Interface plane between front rotating domain and rear rotating domain

(3) Plane 3 - Interface plane between rear rotating domain and stationary domain

(4) Plane 4 - Middle plane of duct diffuser

(5) Plane 5 - End plane of the duct

Figure 6. Plane locations used for plotting.

\subsection{Aerodynamic Performance Analysis of Counter-Rotating Ducted Fan}

Numerical simulations were done for different rotor spacings and duct diffusion angles (see Section 2.1) under five rotating speeds of $1500 \mathrm{rpm}, 1800 \mathrm{rpm}, 2100 \mathrm{rpm}, 2400 \mathrm{rpm}$, and $2700 \mathrm{rpm}$ in hover mode. Figure 7 shows the thrust for the different rotor spacings and duct diffusion angles. The cases with a $0^{\circ}$ duct diffusion angle generated about $9 \%$ higher thrust than the cases with a $6^{\circ}$ duct diffusion angle. The rotor spacing variation had a minimal effect on improving the thrust. The maximum thrust for both duct diffusion angles was observed for the rotor spacing of $200 \mathrm{~mm}$, 
which can also be verified quantitatively from Table 2, where the thrust, thrust coefficient, and the power coefficient are shown for all cases. The thrust increased with increasing rotor spacing up to $200 \mathrm{~mm}$, and then it started to reduce upon further increase of the rotor spacing. The most substantial thrust increment occurred when the rotor spacing increased from $120 \mathrm{~mm}$ to $160 \mathrm{~mm}$. The thrust of the $200 \mathrm{~mm}$ rotor spacing increased by about $1.3-1.5 \%$ compared with that of the $120 \mathrm{~mm}$ rotor spacing. Like the tendency of the thrust results, the maximum thrust coefficient was observed when the rotor spacing was $200 \mathrm{~mm}$. Moreover, the thrust coefficient was higher when the duct diffusion angle was $0^{\circ}$ rather than $6^{\circ}$. However, the power coefficient was at a minimum when the rotor spacing was $120 \mathrm{~mm}$ and, in terms of the diffusion angle, the minimum power coefficient was attained for a $6^{\circ}$ diffusion angle. In summary, from Table 2 , it can be observed that the thrust, thrust coefficient, and power coefficient became higher either when the duct diffusion angle decreased from $6^{\circ}$ to $0^{\circ}$ or when the rotor spacing increased to $200 \mathrm{~mm}$. Figure 8 shows the figure of merit for different rotor spacings and duct diffusion angles. The trend of FOM is similar to the thrust results. The FOM increased by about $1 \%$ with increasing rotor spacing up to $200 \mathrm{~mm}$ from $120 \mathrm{~mm}$, and it reduced gradually upon a further increase of rotor spacing. The duct diffusion angle was more effective than the rotor spacing for improving the FOM. The FOM increased by about $6.7 \%$ when the duct diffusion angle decreased from $6^{\circ}$ to $0^{\circ}$.

Table 2. Thrust, thrust coefficient, and power coefficient of all the counter-rotating ducted fan cases.

\begin{tabular}{|c|c|c|c|c|c|c|}
\hline \multirow{2}{*}{ Duct Diffusion Angle $\left[{ }^{\circ}\right]$} & \multirow{2}{*}{ Rotor Spacing [mm] } & \multicolumn{5}{|c|}{ Thrust [N] } \\
\hline & & \multicolumn{5}{|c|}{ Rotating Speed [rpm] } \\
\hline \multirow{4}{*}{6} & 120 & 49.49 & 71.30 & 97.09 & 126.86 & 160.62 \\
\hline & 160 & 50.18 & 72.27 & 98.40 & 128.58 & 162.72 \\
\hline & 200 & 50.38 & 72.56 & 98.84 & 129.15 & 163.52 \\
\hline & 240 & 50.22 & 72.34 & 98.54 & 128.75 & 162.99 \\
\hline \multirow{4}{*}{0} & 120 & 53.96 & 77.76 & 105.90 & 138.38 & 175.21 \\
\hline & 160 & 54.78 & 78.93 & 107.49 & 140.46 & 177.80 \\
\hline & 200 & 54.95 & 79.17 & 107.84 & 140.89 & 178.36 \\
\hline & 240 & 54.81 & 78.98 & 107.58 & 140.55 & 177.91 \\
\hline \multirow{2}{*}{ Duct Diffusion Angle $\left[{ }^{\circ}\right]$} & \multirow{2}{*}{ Rotor Spacing [mm] } & \multicolumn{5}{|c|}{ Thrust Coefficient } \\
\hline & & \multicolumn{5}{|c|}{ Rotating Speed [rpm] } \\
\hline \multirow{4}{*}{6} & 120 & 0.017335 & 0.017343 & 0.017350 & 0.017357 & 0.017364 \\
\hline & 160 & 0.017576 & 0.017579 & 0.017584 & 0.017592 & 0.017591 \\
\hline & 200 & 0.017646 & 0.017650 & 0.017664 & 0.017671 & 0.017678 \\
\hline & 240 & 0.017589 & 0.017595 & 0.017609 & 0.017616 & 0.017620 \\
\hline \multirow{4}{*}{0} & 120 & 0.018902 & 0.018914 & 0.018924 & 0.018933 & 0.018942 \\
\hline & 160 & 0.019193 & 0.019199 & 0.019208 & 0.019218 & 0.019221 \\
\hline & 200 & 0.019247 & 0.019256 & 0.019272 & 0.019277 & 0.019281 \\
\hline & 240 & 0.019199 & 0.019212 & 0.019226 & 0.019231 & 0.019234 \\
\hline \multirow{2}{*}{ Duct Diffusion Angle $\left[{ }^{\circ}\right]$} & \multirow{2}{*}{ Rotor Spacing [mm] } & \multicolumn{5}{|c|}{ Power Coefficient } \\
\hline & & \multicolumn{5}{|c|}{ Rotating Speed [rpm] } \\
\hline \multirow{4}{*}{6} & 120 & 0.003112 & 0.003103 & 0.003096 & 0.003090 & 0.003085 \\
\hline & 160 & 0.003152 & 0.003143 & 0.003135 & 0.003130 & 0.003125 \\
\hline & 200 & 0.003160 & 0.003151 & 0.003143 & 0.003138 & 0.003133 \\
\hline & 240 & 0.003148 & 0.003139 & 0.003132 & 0.003126 & 0.003122 \\
\hline \multirow{4}{*}{0} & 120 & 0.003327 & 0.003318 & 0.003311 & 0.003305 & 0.003301 \\
\hline & 160 & 0.003371 & 0.003361 & 0.003354 & 0.003349 & 0.003344 \\
\hline & 200 & 0.003372 & 0.003363 & 0.003356 & 0.003351 & 0.003347 \\
\hline & 240 & 0.003362 & 0.003353 & 0.003347 & 0.003341 & 0.003337 \\
\hline
\end{tabular}




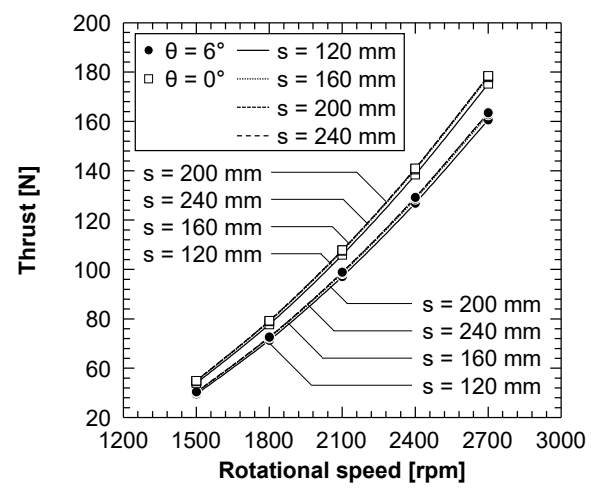

Figure 7. Thrust for different rotor spacings and duct diffusion angles.

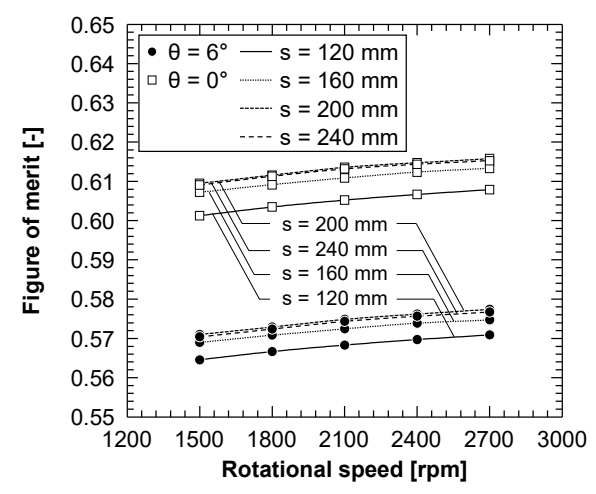

Figure 8. Figure of merit for different rotor spacings and duct diffusion angles.

Since the rotor spacing had a minimal effect on improving the aerodynamic performance of the counter-rotating ducted fan, henceforth, only the results of the cases with rotor spacings of $120 \mathrm{~mm}$ and $200 \mathrm{~mm}$ for $\theta=0^{\circ}, 6^{\circ}$ simulated at the maximum rotational speed of $2700 \mathrm{rpm}$ are discussed. Figure $9 \mathrm{a}-\mathrm{d}$ displays the total pressure contour in Plane 3. See Figure 6 for the location of Plane 3. The negative pressure region close to the duct shows rotor tip leakage loss. As the rotor spacing increases, the thrust increases by about $1 \%$, and, hence, the positive pressure area is extended marginally. The change in the positive pressure area as the rotor spacing increases is not significant. However, when the duct diffusion angle reduces from $6^{\circ}$ to $0^{\circ}$, the magnitude of the positive pressure increases significantly.

Figure 10a-d shows the axial velocity contours in Plane 4 . See Figure 6 for the location of Plane 4. The axial velocity near the hub increases in the radial direction due to the $9 \%$ thrust increase as the duct diffusion angle reduces from $6^{\circ}$ to $0^{\circ}$. As the duct diffusion angle is increased to $6^{\circ}$, the cross-sectional area of the duct is also increased, resulting in a lower axial velocity. The high axial velocity region is widened for an increase in the rotor spacing and a decrease in the duct diffusion angle.

Figure 11a-d shows the axial velocity profiles in the radial direction at different planes. See Figure 6 for the location of the planes. The radial length is normalized with the duct radius. In Figure 11a, the magnitude and profile of the axial velocities located at Plane 1 for all cases are fairly similar. Figure $11 \mathrm{~b}$ indicates that the axial velocity increases at Plane 2 when the rotor spacing is narrow, which is caused by the influence of the rear rotor. The axial velocities in Plane 3 show variation only after the normalized radial length of about 0.7 (see Figure 11c). Moreover, at Plane 3, the magnitude of the axial velocity is higher when $\mathrm{s}=200 \mathrm{~mm}$, due to high thrust. In Figure 11d, the magnitude of the axial velocity at Plane 5 for $\theta=6^{\circ}$ is reduced, resulting from the increased cross-sectional area of the duct. 

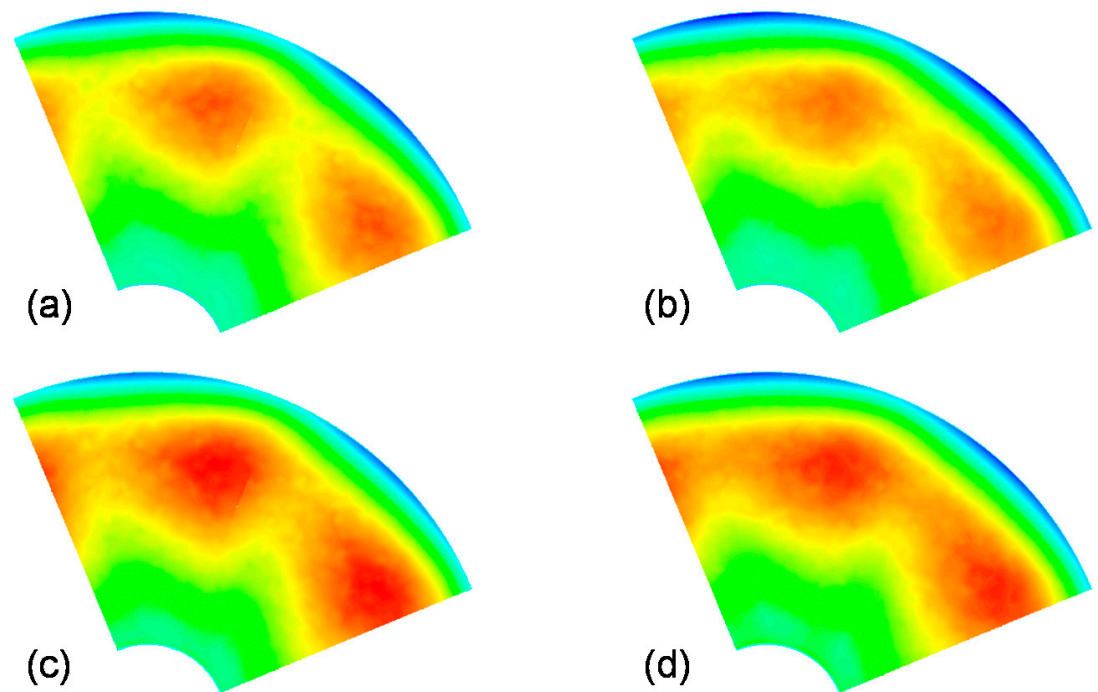

Total pressure $[\mathrm{Pa}]$

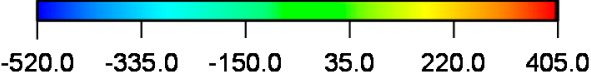

Figure 9. Total pressure contour in Plane 3 at $2700 \mathrm{rpm}$ for: (a) $\theta=6^{\circ}, \mathrm{s}=120 \mathrm{~mm}$; (b) $\theta=6^{\circ}$, $\mathrm{s}=200 \mathrm{~mm} ;(\mathbf{c}) \theta=0^{\circ}, \mathrm{s}=120 \mathrm{~mm}$; and $(\mathbf{d}) \theta=0^{\circ}, \mathrm{s}=200 \mathrm{~mm}$. See Figure 6 for the location of Plane 3.

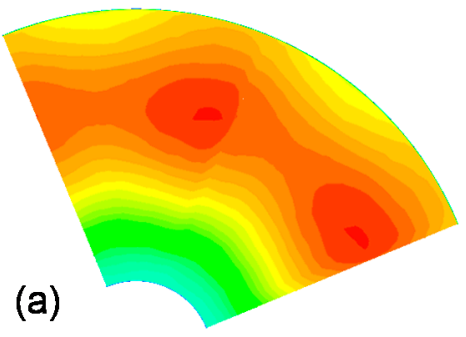

(c)

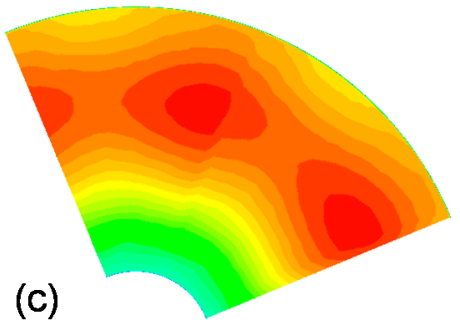

(b)

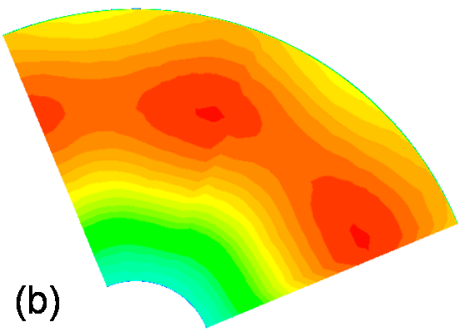

(d)

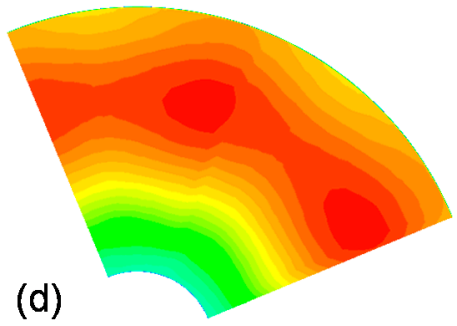

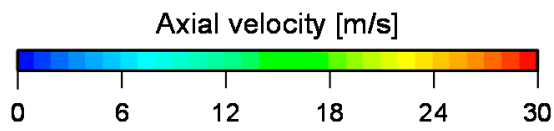

Figure 10. Axial velocity contour in Plane 4 at $2700 \mathrm{rpm}$ for (a) $\theta=6^{\circ}, \mathrm{s}=120 \mathrm{~mm}$; (b) $\theta=6^{\circ}$, $\mathrm{s}=200 \mathrm{~mm} ;(\mathbf{c}) \theta=0^{\circ}, \mathrm{s}=120 \mathrm{~mm}$; and (d) $\theta=0^{\circ}, \mathrm{s}=200 \mathrm{~mm}$. See Figure 6 for the location of Plane 4. 

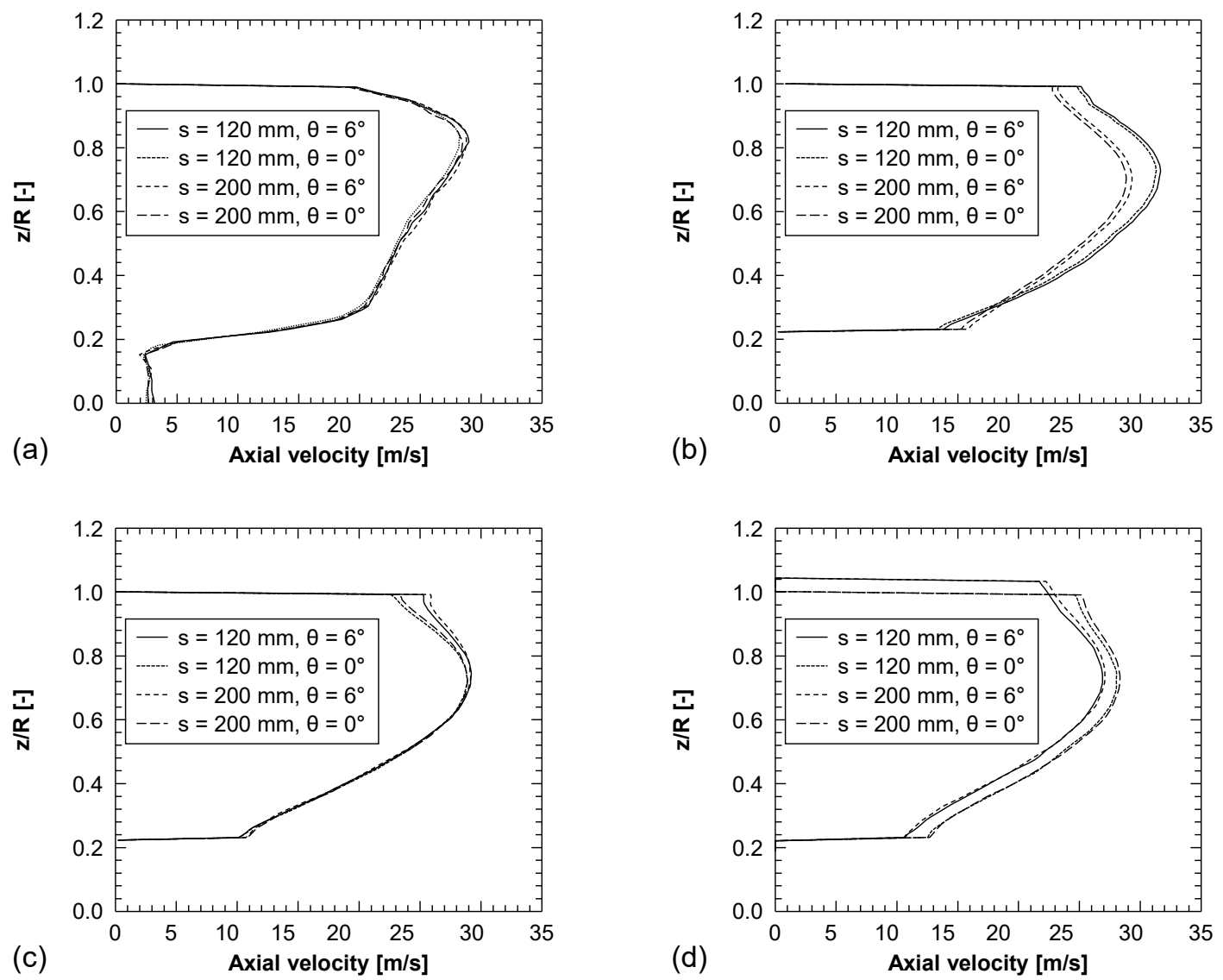

Figure 11. Axial velocity profile along the radial direction at $2700 \mathrm{rpm}$ in (a) Plane 1; (b) Plane 2; (c) Plane 3; and (d) Plane 5. See Figure 6 for the location of the planes.

\section{Conclusions}

The present study explores the aerodynamic performances of a counter-rotating ducted fan in hover mode numerically. The effect of different rotor spacings and duct diffusion angles on the aerodynamic performances is examined. Key findings from this study are as follows:

1. The effect of the relative angle between the front and the rear rotor, due to the usage of the frozen rotor model, is negligible since the variation of thrust for the different relative angles is extremely low.

2. Comparison of the aerodynamic performance parameters for different rotor spacings revealed that the thrust, thrust coefficient, and FOM slightly increases with an increasing rotor spacing up to $200 \mathrm{~mm}$, regardless of the duct diffusion angle. However, the thrust, thrust coefficient, and FOM start to reduce on further increases in the rotor spacing. Conversely, the power coefficient is at a minimum when the rotor spacing is $120 \mathrm{~mm}$.

3. The maximum thrust coefficient is observed when the rotor spacing is $200 \mathrm{~mm}$, and the thrust of the $200 \mathrm{~mm}$ rotor spacing increases by about $1.3-1.5 \%$ compared with the $120 \mathrm{~mm}$ rotor spacing.

4. The duct diffusion angle of $0^{\circ}$ generates about $9 \%$ higher thrust and increases the FOM by $6.7 \%$, compared with the $6^{\circ}$ duct diffusion angle.

5. However, the increase in thrust also increases the power coefficient, which results in increased power consumption. The minimum power coefficient is attained for a $6^{\circ}$ diffusion angle.

6. The duct diffusion angle is highly effective in improving the thrust and FOM of the counter-rotating ducted fan, rather than the rotor spacing. 


\section{Future Work}

The present study discussed the effects of rotor spacing and the duct diffusion angle on the aerodynamic performances of a counter-rotating ducted fan in hover mode. However, to enhance the aerodynamic performance of the UAV, there are still several other design parameters to be considered. Different duct lip shapes and tip clearances could be considered to optimize the counter-rotating ducted fan toward the development of high-performance, efficient UAVs. Moreover, apart from the frozen rotor approach, it is critical to explore various other numerical approaches, such as harmonic analysis techniques, to numerically model the counter-rotating ducted fan.

Author Contributions: Validation, methodology, software, writing-original draft preparation, W.-Y.K.; validation, writing - original draft preparation, S.S.; conceptualization, methodology, supervision, writing-review and editing, S.-M.K. All authors have read and agreed to the published version of the manuscript.

Funding: This research received no external funding.

Conflicts of Interest: The authors declare no conflict of interest.

\section{References}

1. Newman, S. Foundations of Helicopter Flight, 1st ed.; Elsevier: Amsterdam, The Netherlands, 1994.

2. Thwaites, B. Incompressible Aerodynamics, 1st ed.; Oxford University Press: Oxford, UK, 1960.

3. Yilmaz, S.; Erdem, D.; Kavsaoglu, M.S. Performance of a ducted propeller designed for UAV applications at zero angle of attack flight: An experimental study. Aerosp. Sci. Technol. 2015, 45, 376-386. [CrossRef]

4. Bontempo, R.; Manna, M. Effects of Duct Cross Section Camber and Thickness on the Performance of Ducted Propulsion Systems for Aeronautical Applications. Int. J. Aerosp. Eng. 2016, 2016, 9. [CrossRef]

5. Xu, H.Y.; Xing, S.L.; Ye, Z.Y. Numerical Study of Ducted-fan Lip Stall Suppression Based on Inflatable Leading Lip Cell. In Proceedings of the 7th International Conference on Fluid Mechanics, Shandong, China, 24-27 March 2015.

6. Wernicke, K.G.; Wernicke, R.K.; Weisend, N.A., Jr. Inflatable wing leading edge for high lift and deicing. In NASA Tech Briefs; NASA: Washington, DC, USA, 2000; Volume 1.

7. Jiang, Y.; Ye, Z.; Zhan, Z. A method of inflatable leading edge for high lift, deicing and noise reduction. In Proceedings of the 47th AIAA Aerospace Sciences Meeting including The New Horizons Forum and Aerospace Exposition, Orlando, FL, USA, 5-8 January 2009.

8. Graf, W.; Fleming, J.; Ng, W. Improving ducted fan UAV aerodynamics in forward flight. In Proceedings of the 46th AIAA Aerospace Sciences Meeting and Exhibit, Reno, NE, USA, 7-10 January 2008.

9. Jung, S.N.; No, T.S.; Ryu, K.W. Aerodynamic performance prediction of a $30 \mathrm{~kW}$ counter-rotating wind turbine system. Renew. Energy 2005, 30, 631-644. [CrossRef]

10. Vasel-Be-Hagh, A.; Archer, C.L. Wind farms with counter-rotating wind turbines. Sustain. Energy Technol. Assess. 2017, 24, 19-30. [CrossRef]

11. Furukawa, A.; Shigemitsu, T.; Watanabe, S. Performance test and flow measurement of contra-rotating axial flow pump. J. Therm. Sci. 2007, 16, 7-13. [CrossRef]

12. Kim, J.H.; Cho, B.M.; Kim, S.; Kim, J.W.; Suh, J.W.; Choi, Y.S.; Kanemoto, T.; Kim, J.H. Design technique to improve the energy efficiency of a counter-rotating type pump-turbine. Renew. Energy 2017, 101, 647-659. [CrossRef]

13. Shigemitsu, T.; Fukutomi, J.; Okabe, Y. Performance and flow condition of small-sized axial fan and adoption of contra-rotating rotors. J. Therm. Sci. 2010, 19, 1-6. [CrossRef]

14. Toge, T.D.; Pradeep, A.M. Experimental investigation of stall inception of a low speed contra rotating axial flow fan under circumferential distorted flow condition. Aerosp. Sci. Technol. 2017, 70, 534-548. [CrossRef]

15. Srivastava, R.; Sankar, L.N. Efficient hybrid scheme for the analysis of counter-rotating propellers. J. Propuls. Power 1993, 9, 382-388. [CrossRef]

16. Brizzolara, S.; Tincani, E.P.A.; Grassi, D. Design of contra-rotating propellers for high-speed stern thrusters. Ships Offshore Struct. 2007, 2, 169-182. [CrossRef]

17. Ryu, M.; Cho, L.; Cho, J. The effect of tip clearance on performance of a counter-rotating ducted fan in a VTOL UAV. Trans. Jpn. Soc. Aeronaut. Space Sci. 2017, 60, 1-9. [CrossRef] 
18. Han, H.; Xiang, C.; Xu, B.; Yu, Y. Experimental and computational analysis of microscale shrouded coaxial rotor in hover. In Proceedings of the 2017 International Conference on Unmanned Aircraft Systems, Miami, FL, USA, 13-16 June 2017.

19. Corsini, A.; Delibra, G.; Sheard, A.G. A critical review of computational methods and their application in industrial fan design. ISRN Mech. Eng. 2013, 2013, 20. [CrossRef]

20. Akturk, A.; Camci, C. Tip Clearance Investigation of a Ducted Fan Used in VTOL Unmanned Aerial Vehicles-Part I: Baseline Experiments and Computational Validation. J. Turbomach. 2014, 136, 021004. [CrossRef]

21. Ansys CFX 19.1. Theory Guide 2018; ANSYS, Inc.: Canonsburg, PA, USA, 2018.

22. Menter, F.R. Zonal two equation k-w turbulence models for aerodynamic flows. In Proceedings of the 24th Fluid Dynamics Conference, Orlando, FL, USA, 6-9 July 1993.

23. Menter, F.R. Two-equation eddy-viscosity turbulence models for engineering applications. AIAA J. 1994, 32, 1598-1605. [CrossRef]

24. Benim, A.C.; Brillert, D.; Cagan, M. Investigation Into the Computational Analysis of Direct-Transfer Pre-Swirl Systems for Gas Turbine Cooling. In Proceedings of the ASME Turbo Expo 2004: Power for Land, Sea, and Air, Vienna, Austria, 14-17 June 2004.

25. Bohn, D.; Ausmeier, S.; Ren, J. Investigation of the Optimum Clocking Position in a Two-Stage Axial Turbine. Int. J. Rotating Mach. 2005, 3, 202-210. [CrossRef]

26. Chalghoum, I.; Kanfoudi, H.; Elaoud, S.; Akrout, M.; Zgolli, R. Numerical Modeling of the Flow Inside a Centrifugal Pump: Influence of Impeller-Volute Interaction on Velocity and Pressure Fields. Arab. J. Sci. Eng. 2016, 41, 4463-4476. [CrossRef]

Publisher's Note: MDPI stays neutral with regard to jurisdictional claims in published maps and institutional affiliations. 